\title{
Urban Analytics Data Infrastructure:
} Critical SDI for Measuring and Monitoring The National and Local Progress of SDGs

\section{Abbas Rajabifard}

Centre for SDIs and Land Administration, The University of Melbourne, Australia

\section{Soheil Sabri}

Centre for SDIs and Land Administration, The University of Melbourne, Australia

\section{Yiqun Chen}

Centre for SDIs and Land Administration, The University of Melbourne, Australia

\section{Muyiwa Agunbiade}

Centre for SDIs and Land Administration, The University of Melbourne, Australia

\section{Mohsen Kalantari}

Centre for SDIs and Land Administration, The University of Melbourne, Australia

This chapter describes an innovative Spatial Data Infrastructure to support urban analytics and urban research capabilities focused on Australian cities, called Urban Analytics Data Infrastructure (UADI). The UADI provides opportunity for multi-disciplinary, and cross-jurisdictional analytics. The chapter highlights the UADI capabilities to be adopted for deriving the SDG indicators as a response to the UN-GGIM strategic framework 2017 - 2021 technical requirements. 


\subsection{Introduction}

In 2015, the United Nations 2030 Agenda formalised 17 Sustainable Development Goals (SDGs), which consist of 169 targets and 232 indicators. Consequently, the United Nations Committee of Experts on Global Geospatial Information Management (UN-GGIM) aligned their 2017-2021 strategic framework with the 2030 Agenda for Sustainable Development. With the vision of "Positioning Geospatial Information to Address Global Challenges" this strategic framework highlighted several key policies and technical points in their operating principles [12]. The technical points span from agreed standards and methods to integration and interoperability of national information systems as well as information sharing and knowledge transfer. These are important to support the evaluation of SDG indicators at a national level. This is with the assumption that these will enable evidence-based policy making and the development of effective implementation strategies towards achieving the goals set by the 2030 Agenda.

The SDGs have established methodologies that allow the generation of comparable indicators worldwide. Therefore, these methodologies, along with the UN-GGIM operating principles, present opportunities to formalise reusable geospatial tools for producing the indicators. This will allow the UN, and subsequently Member States, to not only compare progress among nations but also to monitor the indicators over time. However, the implementation of the SDG proposed methodologies vary from one jurisdiction and government level to another. This may be due to different terminologies and the subsequent interpretation of their methodologies in various contexts, or the differences in the structural and semantics of the input data used for measuring the indicators. This can result in redundant work for measuring similar indicators and may compromise the comparability of these indicators.

Furthermore, the indicators set forth by governments or other institutions lack transparency of the measurement process and in the case of many existing platforms (e.g. World Council of City Data [WCCD] platform that attempts to present indicators for ISO 37120, urban quality of life), access to such information is limited, affecting the indicator's credibility [14]. These challenges hinder the development of a spatially enabled platform that adopts a set of re-usable geospatial tools for measurement, storage, and effective and transparent communication of the SDG indicators for UN Member States and their respective levels of government. Consequently, customised version of these methodologies are developed to address the subjectivity of indicators and to meet the needs of each jurisdiction. Specifically, this issue can be addressed by a geospatial platform with capability of minimising redundant efforts and encouraging cooperation between different levels of governments, the private sector, academic institutions, and civil society organisations. This is in addi- 
tion to enhancing evidence-based policy making towards achieving the SDGs by providing a repository of a set of transparent and credible indicators.

At the moment, such a geospatial platform that enables the harmonisation of structurally and semantically heterogeneous datasets is lacking. This makes it difficult to work with an ecosystem of re-usable and shared set of user-generated tools for measuring and communicating the SDG indicators. This chapter aims to introduce a Spatial Data Infrastructure (SDI) developed for urban data analytics in Australia [7] and to highlight the capabilities to be adopted for deriving the SDG indicators as a response to the UN-GGIM strategic framework 2017 - 2021 technical requirements.

The next section explains the global indicator framework developed by the Inter-Agency and Expert Group on SDG Indicators (IAEG-SDGs). It explores data and analytics challenges inherent in the framework highlighted by the latest SDGs report. In section three, the chapter introduces Urban Analytics Data Infrastructure (UADI), the motivation for developing such SDI and its components. The section highlights how the UADI is capable of addressing technical requirements indicated in the UN-GGIM strategic framework 2017 - 2021. Consequently, section four explores the possibilities that UADI can contribute to SDGs. Finally, section five provides an account of ways forward in adopting the UADI for deriving SDG indicators in the global context and its implications in achieving SDGs.

\subsection{Global Indicator Framework}

The Inter-Agency and Expert group on SDG (IAEG-SDGs) developed the global indicator framework for the SDGs and targets of the 2030 Agenda for Sustainable Development. This framework, including refinements on several indicators, was agreed upon at the $48^{\text {th }}$ session of the United Nations Statistical Commission held in March 2017. Accordingly, the global framework was adopted by the UN General Assembly on $6^{\text {th }}$ July 2017. This framework is part of the Resolution adopted by the General Assembly on work of the Statistical Commission pertaining to the 2030 Agenda for Sustainable Development ${ }^{1}$.

The global indicator framework emphasised that "Sustainable Development Goal indicators should be disaggregated, where relevant, by income, sex, age, race, ethnicity, migratory status, disability and geographic location, or other characteristics, in accordance with the Fundamental Principles of Official Statistics ${ }^{2} "$. As at April 2018, 232 indicators were listed in this framework. The indicators will be refined annually and will be published in the official website of The United Nations. This will provide information on the develop-

\footnotetext{
${ }^{1}$ Resolution https ://undocs.org/A/RES/71/31371/313

${ }^{2}$ Resolution https ://undocs .org/A/RES/68/26168/261
} 
ment and implementation of indicator frameworks to guide the follow up and review of the 2030 Agenda for Sustainable Development ${ }^{3}$.

While these indicators are defined for national level, several national and international organisations have attempted to localise them. For instance, the Association of Flemish Cities and Municipalities (VVSG), an association of 308 Flemish municipalities and cities, attempted to translate the SDGs at the local level [3]. The VVSG developed tools and guidelines to enable local authorities to develop policies to achieve SDGs. These initiatives require measuring, monitoring, and managing SDG's progress at the local levels. As such, the spatial scope of measuring the SDGs indicators defined at the global framework need to be smaller than what is obtainable at the national level.

From a spatial data point of view, some of the indicators are readily presentable in different geographical boundaries (subject to availability of data). For instance, in target 3.c of SDGs, governments in developing countries are required to "...increase health financing and the recruitment, development, training and retention of the health workforce..." Indicator 3.c.1 intends to measure "Health worker density and distribution". This indicator potentially can be derived and measured in small geographical boundaries of census blocks or administrative boundaries. For other indicators which are not spatial (e.g. indicator 5.5.2 "Proportion of women in managerial positions), they can also be connected to a confined jurisdiction boundary smaller than a state or nation.

It is important to emphasise that the data availability is always a major consideration. As such, a digital platform that is capable of harmonising and standardising the data and analytics tools and then derive SDG indicators is necessary, considering the different data structure and quality. The next section discusses an innovative spatial data infrastructure developed for addressing these challenges.

\subsection{The Urban Analytics Data Infrastructure}

The Urban Analytics Data Infrastructure (UADI) project is a collaborative effort between a consortium of urban research centres across Australia and is funded by the Australian Research Council [7]. The UADI has been developed to enable multi-disciplinary, cross-jurisdiction, national-level analytics of ISO/DIS 37120 "Sustainable development and resilience of communities Indicators for city services and quality of life". It provides a digital infrastructure for urban researchers to overcome current challenges related to data access, integration, analysis and sharing.

Since its development, the UADI has improved the state of urban analytics

\footnotetext{
${ }^{3}$ https://unstats.un.org/sdgs/indicators/indicators-list/
} 
in Australia, and capitalised on previous urban data initiatives, for example the Australian Urban Research Infrastructure Network (AURIN). This has provided opportunities to add more value to the existing initiatives. It also provides the capability to shift the current urban research and planning landscape towards one that is more consistent across jurisdictions. It builds up the requisite intellectual capital to support evidence-based decision-making that transcends traditional disciplinary domains.

In addition, the UADI facilitates analytics tool sharing and provides metadata for both data and tools. These capabilities in the UADI are developed to increase the reliability, trustworthiness, and useability of data, tools, and output information. As such, this Spatial Data Infrastructure (SDI) attempts to address several challenges related to the data and deriving city indicators recently raised by scholars worldwide [4, 14].

As a digital data infrastructure, the UADI enables the integration, harmonisation, connectivity and scalability of multi-source urban datasets. As applied, for an example, in the analysis of urban density, this infrastructure was able to integrate data related to population, building footprints, and land use, which could be used to compare different urban densities (e.g. residential/commercial built-up area per capita, and publicly available open space per capita) in local authorities across Metropolitan Melbourne. The infrastructure developed a new ontological framework [1] and a dictionary to underpin the next generation of data driven modeling and decision-support tools to enable smart, sustainable, productive, and resilient cities.

UADI DASHBOARD

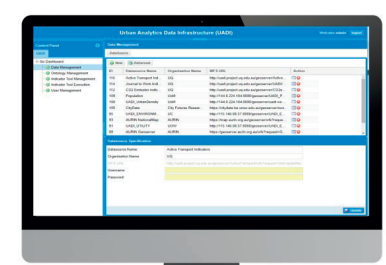

http://apps.csdila.ie.unimelb.edu.au/uadi/index.html

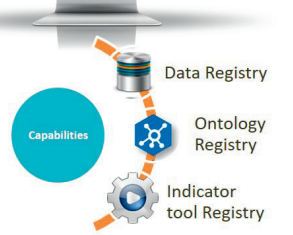

UADI PORTAL

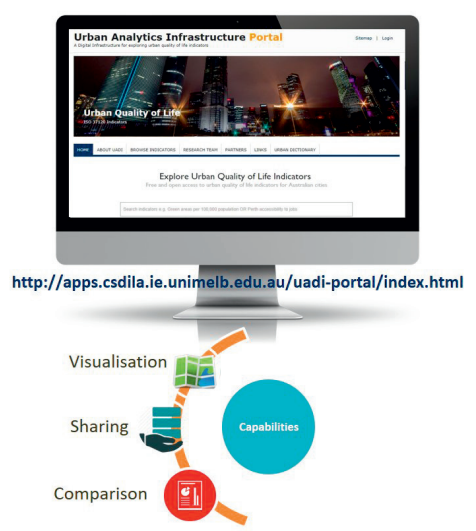

\section{FIGURE 15.1}

The UADI components and capabilities

The main objectives in developing UADI are as follows: 
1. To provide an underlying framework for harmonisation and integration of urban data by adopting the ISO 37120 and ISO 19115 standards.

2. To develop core system capabilities including data registry, integration and access, as well as analytics tools registry, execution and publishing through web APIs (Application Programming Interfaces) by adopting the OGC (Open Geospatial Consortium) standards.

3. To develop an integrated platform and web-portal to visualise and evaluate the cross-jurisdictional and cross-domain performances.

4. To facilitate open access to those datasets currently accessible through the AURIN Data Hubs and any new open data sets through the development of open access APIs.

The UADI addresses challenges such as those associated with data access, data integration, and the use of varying terminology between disciplines. This infrastructure is comprised of two main components (Figure 15.1):

1. UADI Dashboard: The aim of this component is to allow users to access, integrate, and semantically enrich the data as well as manage and execute their own or others' analytics tools. Users can also preview and publish the results of the analytics in the dashboard.

2. UADI Portal: The aim of this portal is to enable public users to discover, access, explore, and compare urban quality of life indicators calculated by a variety of contributors from different sectors such as research and development, government, and the private sector.

The core capability of the UADI is using ontology that consists of one (or more) upper-level and domain ontologies, describing the generic (e.g. space and time) or specialised concepts pertinent to one or more domains of knowledge [2]. As an example, one of the upper-level ontologies developed in UADI is for Austrian Statistical Geography Standard (ASGS), which determines the relationships of statistical and administrative geographical boundaries across Australia (Figure 15.2). Furthermore, using METHONTOLOGY [1], a number of application ontologies for urban density and urban accessibility measurements were developed.

In addition to the definition of concepts, their relationships are also defined by ontology in the UADI. Therefore, the mapping between any data and its attribute in a dataset to one or many concepts within the ontology can be used to describe the dataset for discovery, and also data integration purposes $[1]$.

The data in the UADI model refers to the datasets that are available from the data providers through standard web services and over the Internet. The data can be spatial or non-spatial and can be structured and non-structured. This data should be exposed via the data custodians to UADI through standardised OGC protocols (e.g. Web Feature Services) [11]. These services can 
then be registered using their metadata and via the data catalogue component in the UADI.

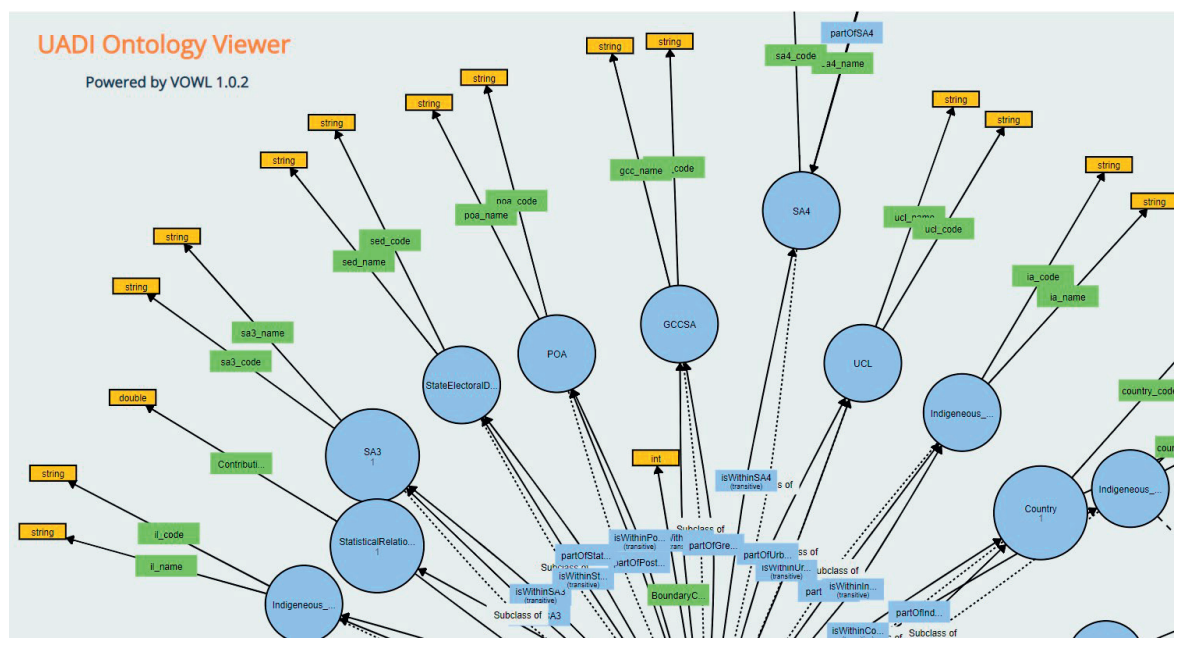

\section{FIGURE 15.2}

An example of using VOWL (v1.0.2) to show the concepts (blue circles), properties (green blocks) and relations (blue blocks) defined in the ASGS Ontology. The subclass of a concept is represented in dashed arrows.

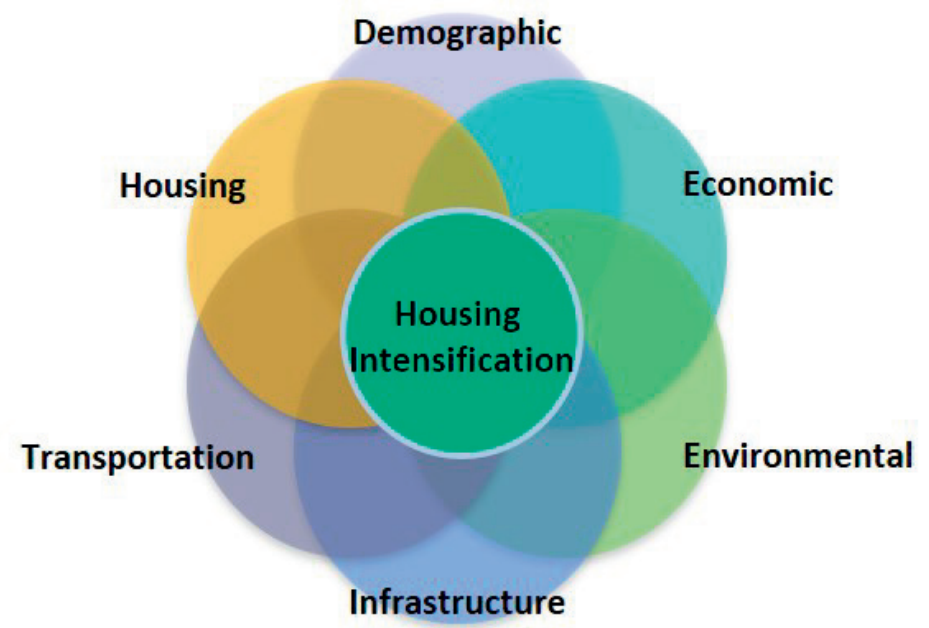

\section{FIGURE 15.3}

Housing intensification; a multi-domain urban planning approach.

This infrastructure is a smart technology that can be used to generate 
indicators for decision makers, such as urban density [1] and accessibility [10]. For this purpose, the UADI encapsulates and registers each indicator tool as a Web Processing Service (WPS) [11] endpoint, which can then be executed by users with various parameters as inputs for deriving the indicators. This functionality enables organisations to conduct more advanced multi-domain spatial analyses such as urban heat island (UHI) and housing intensification. For instance, as can be seen in Figure 15.3, housing intensification is an urban planning approach, which involves several integrated and overlapping domains which need to be analysed in an infrastructure that can integrate data from multi-domains.

Using the UADI, it is also possible to integrate data to do transparent and comparable analysis among different cities. Figure 15.4 shows the comparison of spatial distribution pattern for one of the urban density parameters (Plot Factor: The ratio of private land areas to land available for public use, distinguished by functionality and accessibility) in two local authorities of Moreland (an inner-suburb) and Whitehorse (a middle-suburb) in Metropolitan Melbourne. Figure 15.4 also shows how the results of analyses are normalised (the two lower maps) to facilitate the comparisons.

\subsection{UADI's Contribution to SDGs}

The literature on city indicators has highlighted several challenges about transparency, reliability, and usefulness of the indicators [4]. As explained in the first section, the lack of data provenance and uncertainty in indicator measurement process in the case of existing platforms limits the credibility of indicators. These challenges apply to SDG indicators as well as there are specific local conditions for one region which may have not been the case in others. So how can countries register the process based on which indicators are derived?

In 2016, when the transforming plan from Millennium Development Goals (MDGs) to SDGs was prepared by the member states, several limitations about data and decision making processes were highlighted. One of the bottlenecks was the lack of quality data to enable regular monitoring and support evidence-based decision making. Accordingly, international agencies including UNDP, World Bank Group, and UN-Habitat suggested using real-time data, adopting geospatial data, strengthening statistical capacity, utilising new technologies, changing the methods of data collection and dissemination, developing global standards for integrated statistical systems, and promoting open data $[13,5]$.

As such, in order to measure, monitor and compare the SDG's progress by the UN member states, a spatially enabled information decision making platform is critical. Such a platform should enable the member states to derive 

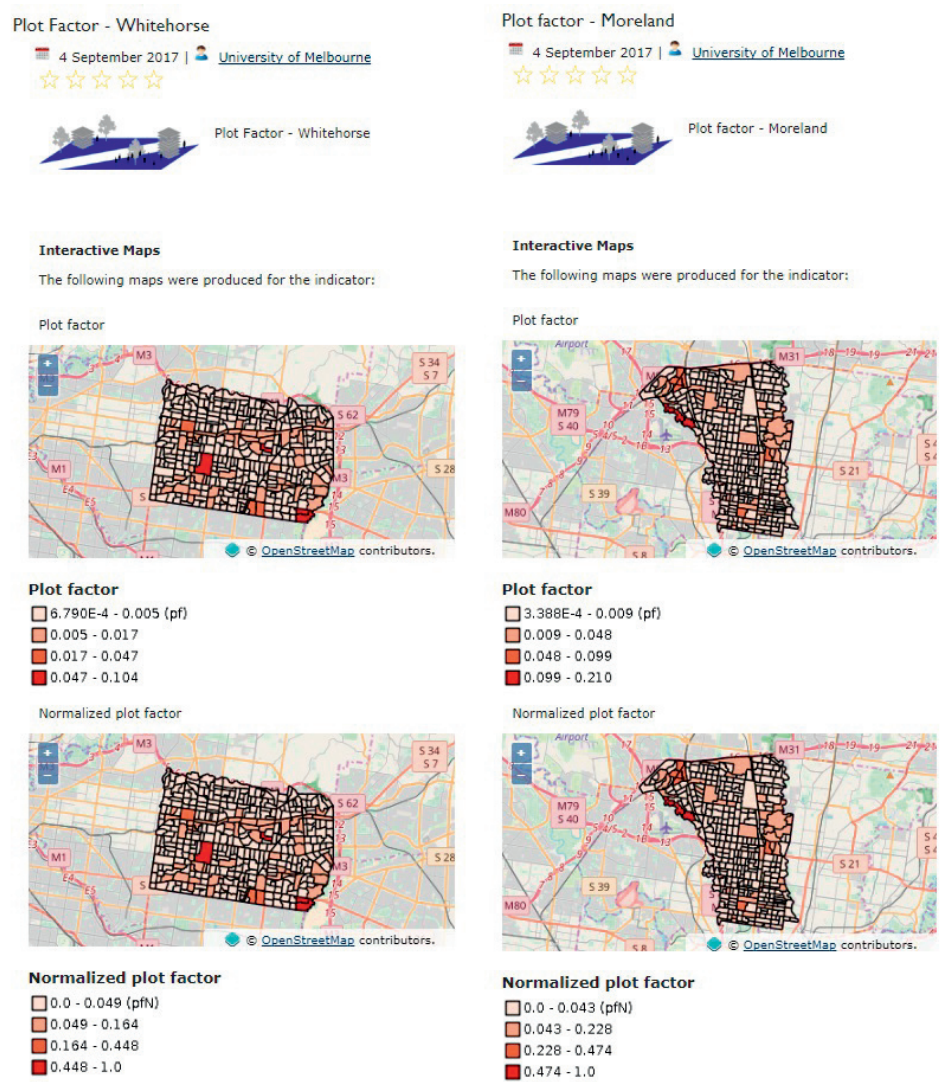
Plot factor
$\square 3.388 \mathrm{E}-4 \cdot 0.009$ (pf)
$\square 0.009-0.048$
$0.048-0.099$
$0.099-0.210$

Normalized plot factor

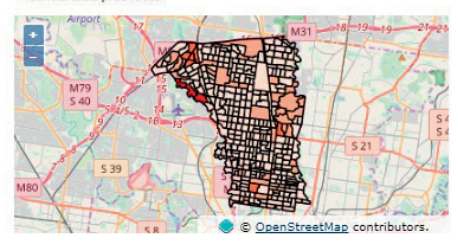

Normalized plot factor

$\square 0.0-0.043(\mathrm{pfN})$

$0.043-0.228$

$\square 0.228-0.474$

$0.474-1.0$

\section{FIGURE 15.4}

Spatial comparison of plot factor in two local authorities in metropolitan Melbourne.

their indicators through data access and integration facility using reliable and replicable tools in order to visualise and share the outcomes of the SDG indicators (Figure 15.5). The UADI, as explained in the previous section, is a digital infrastructure with the capability to meet the SDG's progress management requirements in conceptual framework presented in Figure 15.5. Furthermore, the UADI is potentially an enabler to progress the UN-GGIM's strategic framework. It is also designed to operate the principles that allow the formalisation of re-usable geospatial tools in capacity building, thereby enabling the UN and member states to compare, monitor and manage SDG progress.

The UADI's capability in registering spatial and non-spatial data enables deriving SDG indicators that are non-spatial as well. In some cases, it is possible to connect the non-spatial indicators to a certain geographical boundary. 


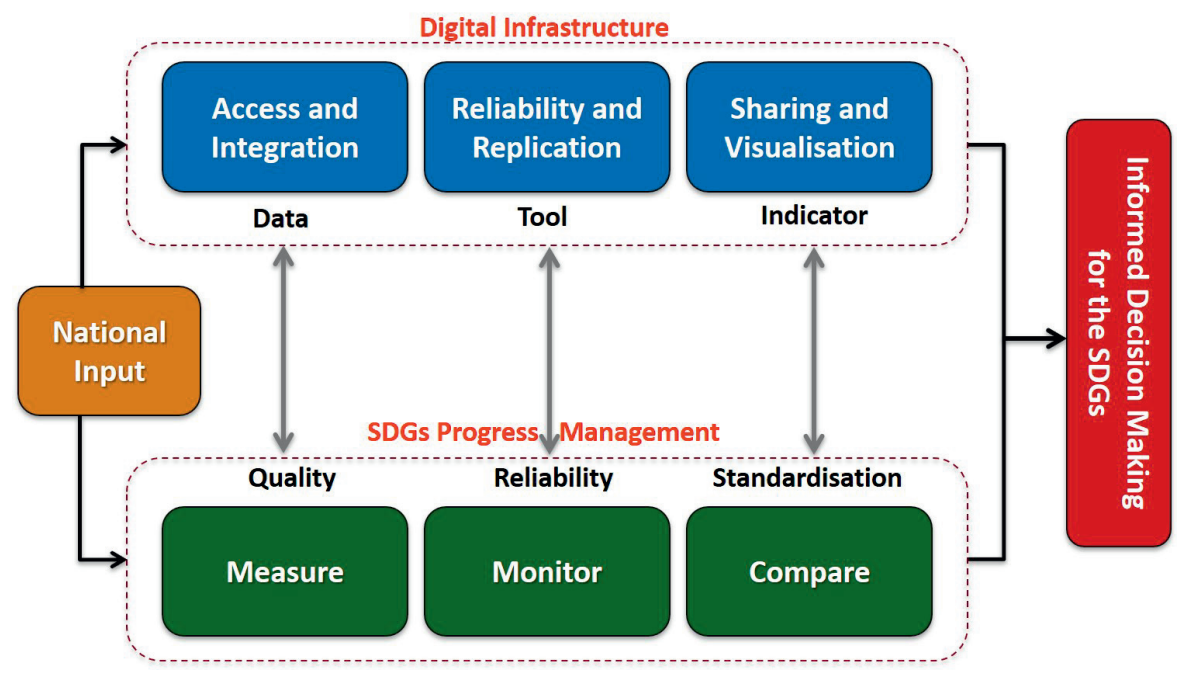

\section{FIGURE 15.5}

Conceptual framework for a digital infrastructure enabling the SDG's progress management.

As an example, as part of target 1.4 of the SDGs, governments are required to "...ensure all men and women have equal rights to economic resources, and access to basic services, and control over land and other forms of property..." Indicator 1.4.2 intends to measure "proportion of total adult population with secure tenure rights to land, with legally recognised documentation and who perceive their rights to land as secure...". Subject to the availability of data, this SDG indicator can be attributed to a particular census or administrative boundary. As a result, regarding the 1.4.2 indicator, UN Member States can provide a better understanding of the rate of progress in a spatially and temporally visualised fashion, which identifies the deficiencies that require further improvement at local, state, and national government levels.

In addition, the UADI enables the evaluation for potential future scenarios. This capability can additionally facilitate strategic planning and informed decision making for optimal solutions amongst various alternative options. This capability of UADI enables defining use cases such as land development, housing affordability, emergency and energy efficiency, and inclusive infrastructure development (e.g. transport, telecommunications, and other utilities). The use cases along with indicators will enable different government levels to localise the implications of SDGs (Figure 15.6). It is also important to improve the UADI by using the live data (e.g. sensor network), and big data for advanced analytics. 


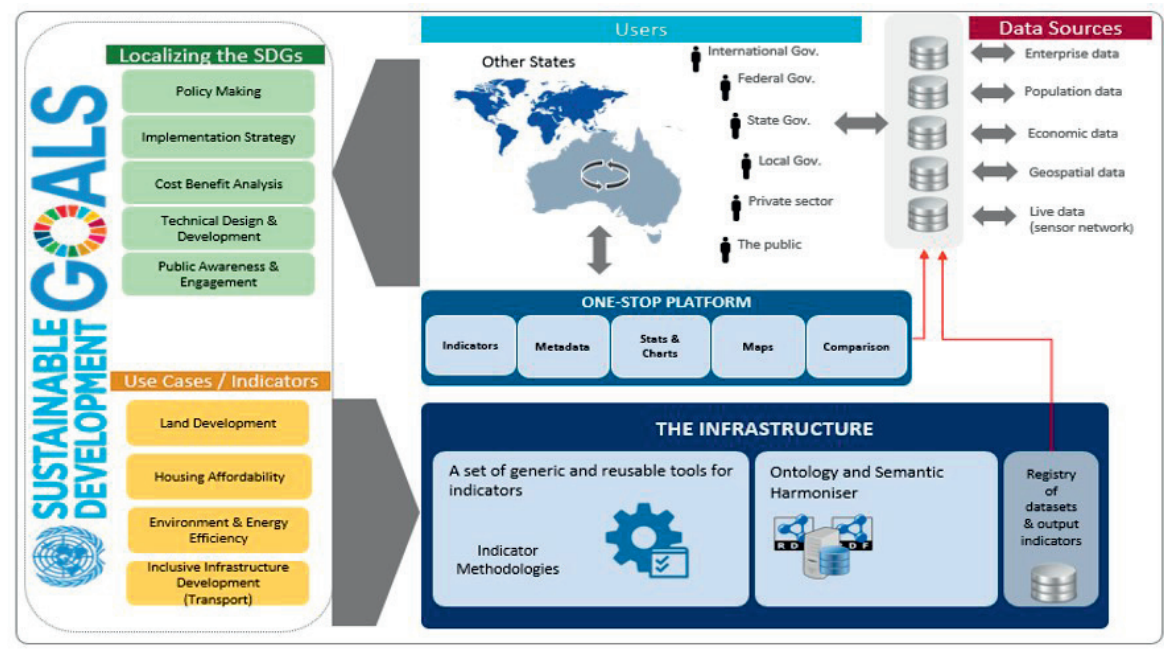

FIGURE 15.6

Potential contribution to SDGs and localising the SDG indicators.

\subsection{Discussion and Conclusion}

This chapter discussed how the development of national indicators for the SDGs and localising the 2030 Agenda for Sustainable Development require a digital infrastructure that can facilitate the registration and sharing of indicators locally and globally. The chapter then introduced a spatial data infrastructure (SDI) that enables the harmonisation of structurally and semantically heterogeneous datasets to work with an ecosystem of re-usable and shared set of user-generated tools for measuring and communicating the SDGs indicators.

The chapter has also discussed the capabilities that UADI platform offers for deriving the SDG indicators as a response to the UN-GGIM strategic framework 2017 - 2021 technical requirements. The UN-GGIM can facilitate the adoption of such a digital infrastructure for member states to register new indicators related to land administration, disaster risk reduction, food security, and the implementation of standards in order to measure and monitor the inclusive progress of the SDGs.

One of the major advantages of the UADI is its capability to spatially visualise the indicators, which help to benchmark and compare different jurisdictions. Each local, state, and national government can also compare their own progress in SDG management by investigating the changes of SDG indicators through time.

The chapter has also shown that the UADI system offers several innova- 
tions, which highlight future research directions for new developments in areas such as:

- Ontology and its role in national and international linked data projects. The UADI system has already addressed several challenges in the Australian National Linked Data project, which put Australia at the forefront of open data and semantic web development.

- Formalising the Open Geospatial Consortium (OGC) smart city framework [6] and a new agenda for incorporating sensor data, crowd-sourcing volunteered geographic information data (VGI) [9] for real time analytics in deriving SDG indicators.

- Adding more use cases related to sustainability, including liveability [8] and quality of life indicators facilitating the localisation of SDGs. The use cased will help in local policy making, refining strategies and public awareness and engagement for implementing SDGs.

\section{Acknowledgment}

The UADI project is supported by an Australian Research Council Linkage Infrastructure Equipment Facilities (ARC LIEF) grant (LE160100174). The authors would like to acknowledge the contribution of Maryam Rabiee in developing Figures 5 and 6 and Sam Amirebrahimi in developing Figure 6.

\section{Bibliography}

[1] Yiqun Chen, Soheil Sabri, Abbas Rajabifard, and Muyiwa Elijah Agunbiade. An ontology-based spatial data harmonisation for urban analytics. Computers, Environment and Urban Systems, 72:177-190, 2018.

[2] Gilles Falquet, Claudine Métral, Jacques Teller, and Christopher Tweed. Ontologies in urban development projects. Springer Science \& Business Media, 2011.

[3] Bart Van Herck, Valentijn Vanoeteren, and Katrin Janssen. Local indicators for the 2030 agenda (sustainable development goals), 2019. Brussels, Belgium.

[4] Rob Kitchin and Gavin McArdle. Urban data and city dashboards: Six key issues. 2016. 
[5] United Nations. The Millennium Development Goals Report 2015. 2015.

[6] George Percivall, C Ronsdorf, S Liang, D McKenzie, and L McKee. Ogc smart cities spatial information framework. OGC Internal reference, (14115), 2015.

[7] Abbas Rajabifard, Serene Ho, and Soheil Sabri. Urban analytics data infrastructure: Critical sdi for urban management in australia. SMART WORLD, page 95, 2016.

[8] S Sabri, S Ho, and A Rajabifard. Implementing the '20-minute neighbourhood': Leveraging new spatial data and technologies to support planning for a sustainable metropolitan melbourne. Healthy Future Cities; Sun, SH, Wenqi, L., Eds, pages 502-517, 2016.

[9] Soheil Sabri, Abbas Rajabifard, Serene Ho, Sam Amirebrahimi, and Ian Bishop. Leveraging vgi integrated with 3d spatial technology to support urban intensification in melbourne, australia. Urban Planning, 1(2):3248, 2016.

[10] Yuchao Sun, Gary McCarney, Soheil Sabri, Sam Amirebrahimi, Muyiwa Agunbiade, Yiqun Chen, Azadeh Keshtiarast, Mohsen Kalantari, Callan Bright, Doina Olaru, et al. A flexible accessibility analysis tool for enhanced urban analytics. In Proceedings of the Australasian Transport Research Forum, Auckland, New Zealand, pages 27-29, 2017.

[11] Chet Bing Tan, David A McMeekin, Geoff West, and Simon Moncrieff. Ciao-wps-utilizing semantic web (web 3.0) techniques to assist in the automatic orchestration of geospatial processes and datasets. In International Conference on Geographical Information Systems Theory, Applications and Management, pages 32-48. Springer, 2016.

[12] UN-GGIM. Strengthening geospatial information management. Technical report, 2017.

[13] UNDP and World Bank Group. Transitioning from the mdgs to the sdgs, 2016.

[14] Yetian Wang and Mark S Fox. Consistency analysis of city indicator data. In International Conference on Computers in Urban Planning and Urban Management, pages 355-369. Springer, 2017. 